\title{
A model-based analysis: what potential could there be for a S. aureus vaccine in a hospital setting on top of other preventative measures?
}

\author{
Cosmina Hogea ${ }^{1 *}$, Thierry Van Effelterre ${ }^{2}$ and Adrian Cassidy ${ }^{2}$
}

\begin{abstract}
Background: Over the past decade, there has been sustained interest and efforts to develop a S. aureus vaccine. There is a need to better evaluate the potential public health impact of $S$. aureus vaccination, particularly given that preventative measures exist to reduce infection. To our knowledge, there is no previous work to assess the potential of a S. aureus vaccine to yield additional MRSA infection reduction in a hospital setting, on top of other preventative measures that already proved efficient.
\end{abstract}

Methods: The main objectives were to propose a versatile simulation framework for assessing potential added benefits of a hypothetical $S$. Aureus vaccine in conjunction with other preventative measures, and to illustrate possibilities in a given hospital setting. To this end, we employed a recently published dynamic transmission modelling framework that we further adapted and expanded to include a hypothetical $S$. aureus vaccination component in order to estimate potential benefits of vaccinating patients prior to hospital admission.

Results: Model-based projections indicate that even with other hygiene prevention measures in place, vaccination of patients prior to hospital admission has the potential to provide additional reduction of MRSA infection. Vaccine coverage and vaccine efficacy are key factors that would ultimately impact the magnitude of this reduction. For example, in an average case scenario with 50\% decolonization, 50\% screening and 50\% hygiene compliance level in place, S. aureus vaccination with $25 \%$ vaccine coverage, $75 \%$ vaccine efficacy against infection, and $0 \%$ vaccine efficacy against colonization, may lead to $12 \%$ model-projected additional reduction in MRSA infection prevalence due to vaccination, while this reduction could reach $37 \%$ for vaccination with $75 \%$ vaccine coverage and $75 \%$ vaccine efficacy against infection in the same average case scenario.

Conclusions: S. aureus vaccination could potentially provide additional reduction of MRSA infection in a hospital setting, on top of reductions from hygiene prevention measures. The magnitude of such additional reductions can vary significantly depending on the level of hygiene prevention measures in place, as well as key vaccine factors such as coverage and efficacy. Identifying appropriate combinations of preventative measures may lead to optimal strategies to effectively reduce MRSA infection in hospitals.

Keywords: Mathematical modelling, MRSA, Hospital infection, Infection reduction, Vaccination, Preventative measures

\footnotetext{
* Correspondence: cosmina.s.hogea@gsk.com

'GlaxoSmithKline Vaccines, 2301 Renaissance Blvd Ste RN0510, King of Prussia, PA, USA

Full list of author information is available at the end of the article
}

\section{Biomed Central}

(c) 2014 Hogea et al.; licensee BioMed Central Ltd. This is an Open Access article distributed under the terms of the Creative Commons Attribution License (http://creativecommons.org/licenses/by/2.0), which permits unrestricted use, distribution, and reproduction in any medium, provided the original work is properly credited. 


\section{Background}

Staphylococcus aureus (S. aureus) is an important opportunistic pathogen and the aetiological agent of a wide range of infections, from mild skin and soft tissue infections to bactaeremia complicated by endocarditis, pneumonia, metastatic infections, sepsis or toxic shock syndrome [1]. S. aureus is an ubiquitous pathogen, part of the human microbiological flora. It persistently and asymptomatically colonizes up to $20-30 \%$ of humans and intermittently colonizes $50-60 \%$ [1].

Following the introduction of methicillin in 1959, various methicillin-resistant S. aureus (MRSA) clones (resistant to all currently available $\beta$-lactam antibiotics) emerged and spread worldwide [2]. Since the 1960s, when the first outbreak of healthcare-associated MRSA (HA-MRSA) was reported [3], HA-MRSA clones have been recognized as a leading cause of nosocomial infections in the United States and around the world $[4,5]$. A worldwide increase in the number of infections, together with the emergence of community-associated MRSA (CA-MRSA) in the late 1990s and its expansion into hospital settings [6], has increased the burden of MRSA infections [1]. Prevention of MRSA infections has become a goal for public health agencies and policy makers [7-9].

Several studies, the majority of which were conducted in individual or small groups of facilities, have recently shown that the rates of MRSA infections decreased following implementation of MRSA prevention strategies [10-15]. A number of studies have assessed the impact of using a combination of different prevention strategies ('bundle' measures), including universal surveillance for MRSA, contact precautions, hand hygiene and institutional culture change programs [11,12,15-18]. For example, a clusterrandomized trial evaluating the effect of active surveillance and expanded use of barrier precautions for MRSA and vancomycin-resistant Enterococcus (VRE) compared to 'existing procedures' during a 6-month intervention period, did not find that the intervention was more effective than existing practice in reducing the incidence of colonization or infection with MRSA or VRE [16]. However many studies have shown a positive effect of implementing the bundle measures in endemic settings [11,12,15,17,18]. An observational study on the effect of MRSA bundle measures, implemented in 2007 in Veterans Affairs hospitals throughout the United States, reported 62\% and 45\% decreases in rates of HA-MRSA infections in ICUs (Intensive Care Units) and non-ICUs following a 3-year intervention period [18].

Although the bulk of the evidence suggests that bundle measures are efficient towards reducing the incidence of MRSA nosocomial infections, the mortality, morbidity and costs associated with these infections remain high. $S$. aureus infections are the leading cause of hospitalization for surgical drainage of pus in children, of bacteremia in persons aged $>65$ years, and of prosthetic device and intravascular line infection [19]. In this context, during the last decade there has been a sustained interest and effort [19-22] to develop an effective vaccine against $S$. aureus [19], and several vaccine candidates are in early stage development [22].

Given the many challenges of developing a $S$. aureus vaccine [22], a natural timely question arises regarding its potential added value to positively impact the burden of MRSA infection at the hospital level. To address this question, we employ here a versatile dynamic transmission modelling framework complementary to, and expanding that used in D'Agata et al. [23], with the aim of assessing and quantifying the potential impact of a $S$. aureus vaccine in conjunction with hygiene prevention measures on reducing MRSA infection in a hospital setting. The main objectives were to both propose an adequate simulation framework for assessing potential added benefits of a hypothetical $S$. aureus vaccine in conjunction with other preventative measures and illustrate the spectrum of possibilities with a few relevant scenarios in a given hospital setting. The analyses we present here were not intended to be exhaustive, but rather to serve as an illustrative starting point.

\section{Methods}

To our knowledge, this study is the first to evaluate if and how $S$. aureus vaccination prior to hospital admission may further reduce MRSA infection in a given hospital setting; particularly since hygiene prevention measures can be quite effective.

\section{Mathematical modelling overview}

For our first attempt to estimate potential benefits of vaccinating patients prior to hospital admission, we chose to start by employing a recently published dynamic transmission modelling framework [23,24], which we further adapted and expanded to meet our specific objectives.

One of the main rationales for building a vaccination component on top of an existing published model [23,24] was that baseline estimates for all the model parameters were provided for a given hospital setting in the US. In line with the previously published work [23,24], the present framework is based on a dynamic transmission model for MRSA only, further split into HA-MRSA and CA-MRSA. Methicillin sensitive S. aureus (MSSA) is not included. The original base model structure, which explicitly accounts for HA-MRSA and CA-MRSA, with different corresponding baseline model parameters (Table 1), was preserved as actual and appropriate to account for the realities of MRSA transmission in a hospital setting in the US. The explicit inclusion of HA-MRSA and CA-MRSA is adequate here as CA-MRSA is a growing problem in US hospitals, with CA-MRSA strains genetically distinct 
Table 1 Model parameters, with baseline values imported from [23,24]

\begin{tabular}{|c|c|c|}
\hline Parameter & Symbol & Baseline value \\
\hline Total no. of patients & N & 400 \\
\hline Fraction of patients colonized with CA-MRSA upon admission & $\lambda_{C \subset}^{\text {baseline }}$ & 0.03 \\
\hline Fraction of patients colonized with HA-MRSA upon admission & $\lambda_{C H}^{\text {baseline }}$ & 0.07 \\
\hline Fraction of patients infected with CA-MRSA upon admission & $\lambda_{I C}^{\text {baseline }}$ & 0.005 \\
\hline Fraction of patients infected with HA-MRSA upon admission & $\lambda_{\mathbb{H}}^{\text {baseline }}$ & 0.0017 \\
\hline Average length of stay susceptible patients & $1 / \gamma_{\mathrm{s}}$ & 5 days \\
\hline Average length of stay patients colonized with CA-MRSA & $1 / \gamma_{\subset c C}$ & 5 days \\
\hline Average length of stay patients colonized with HA-MRSA & $1 / \mathrm{Y}_{\mathrm{CH}}$ & 7 days \\
\hline Average length of stay patients infected with CA-MRSA & $1 / \gamma_{I C}$ & 10 days \\
\hline Average length of stay patients infected with HA-MRSA & $1 / \gamma_{1 H}$ & 18 days \\
\hline CA-MRSA colonized-to-susceptible patient effective transmission rate & $\beta_{C C}^{\text {baseline }}$ & 0.36 day $^{-1}$ \\
\hline HA-MRSA colonized-to-susceptible patient effective transmission rate & $\beta_{C H}^{\text {baseline }}$ & 0.27 day $^{-1}$ \\
\hline CA-MRSA infected-to-susceptible patient effective transmission rate & $\beta_{l c}^{\text {baseline }}$ & 0.09 day $^{-1}$ \\
\hline HA-MRSA infected-to-susceptible patient effective transmission rate & $\beta_{\mathbb{H}}^{\text {baseline }}$ & 0.07 day $^{-1}$ \\
\hline \multirow[t]{2}{*}{ Infection rate in CA-MRSA colonized patients } & $\varphi_{C}$ & $0.1 \gamma_{\subset C}$ \\
\hline & & (10\% per day of hospital stay) \\
\hline \multirow[t]{2}{*}{ Infection rate in HA-MRSA colonized patients } & $\varphi_{H}$ & $0.1 \gamma_{\mathrm{CH}}$ \\
\hline & & (10\% per day of hospital stay) \\
\hline \multirow[t]{2}{*}{ Death rate of CA-MRSA infected patients } & $\delta_{C}$ & $0.033 \gamma_{I C}$ \\
\hline & & (3.3\% per day of hospital stay) \\
\hline \multirow[t]{2}{*}{ Death rate of HA-MRSA infected patients } & $\delta_{H}$ & $0.2 \gamma_{\mathbb{H}}$ \\
\hline & & ( $20 \%$ per day of hospital stay) \\
\hline \multirow[t]{2}{*}{ Cure rate of CA-MRSA infected patients } & $\tau_{C}$ & $0.967 Y_{K}$ \\
\hline & & ( $96.7 \%$ per day of hospital stay) \\
\hline \multirow[t]{2}{*}{ Cure rate of HA-MRSA infected patients } & $\tau_{H}$ & $0.8 \gamma_{\mathbb{H}}$ \\
\hline & & (80\% per day of hospital stay) \\
\hline
\end{tabular}

from HA-MRSA strains and thought to have evolved separately [25]. Such features allow for potential differential transmission aspects to be taken into consideration, thus enabling more realistic estimations of the overall reduction of MRSA infection in hospital settings following implementation of different interventions. The proposed modelling framework also readily enables consideration of potentially different values for vaccine-related parameters for HA-MRSA strains versus CA-MRSA strains, which may be an important aspect for future vaccine evaluations, in light of underlying genotypic differences.

In the analyses presented here, for the time being we have considered identical vaccine-related parameters for both HA-MRSA and CA-MRSA so as to reduce the number of possible combinations of parameters.

The model's setting is a large tertiary hospital in the US, with a fixed capacity of 400 beds, and approximately 25000 admissions per year [23]. For simplicity and consistency, and in the absence of hospital occupancy data over time, we assumed that the hospital is fully occupied at all times, throughout our simulations. We consequently varied the daily number of admissions (typically 60-80 patients/day in our simulations) to ensure full hospital capacity. In this simplified framework, we hypothesized that patients are adequately vaccinated with a $S$. aureus vaccine before hospital admission (e.g. planned hospitalization, vaccination of high-risk individuals), in an optimal timeframe (e.g., few weeks) to yield a protective immune response for the duration of the hospital stay. Similar to the baseline case from the original paper [23], we assumed a constant in-flow of colonized patients into the hospital corresponding to $10 \%$ (fixed) of the daily admission numbers.

\section{Model structure}

The detailed model structure including a hypothetical $S$. aureus vaccination component is shown in Figure 1, resulting in a highly versatile modelling framework, with enhanced options for conducting differential analyses. We employ a dynamic transmission model with two components, "Unvaccinated" and "Vaccinated", respectively. Each 


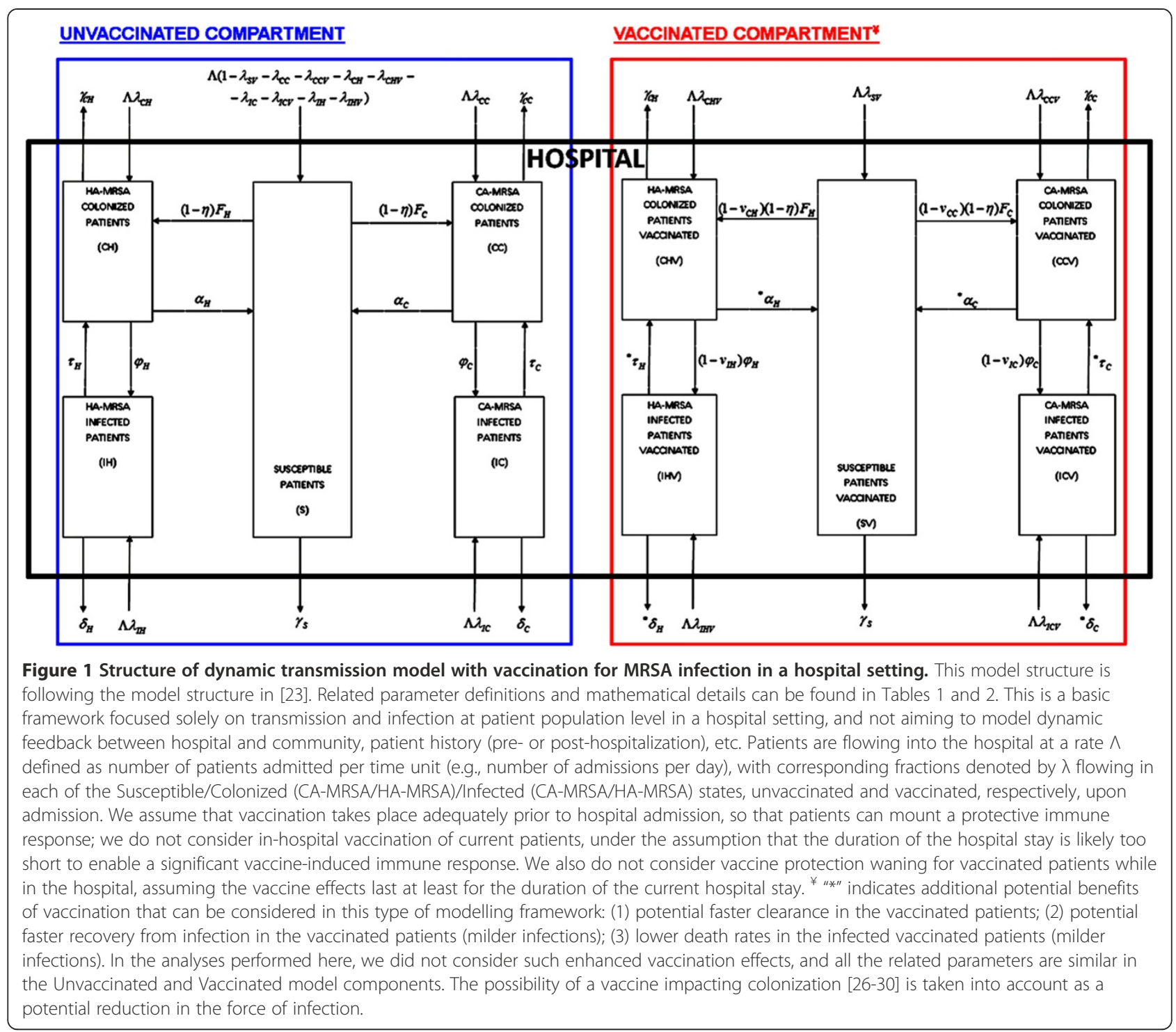

component contains five distinctive states: susceptible $(S)$ patients, patients colonized with HA-MRSA ( $\mathrm{CH}$ patients), patients colonized with CA-MRSA ( $C C$ patients), patients infected with HA-MRSA ( $\mathrm{H}$ patients) or infected with CA-MRSA (IC patients). The additional "V" notation here is used for the corresponding vaccinated states. Patients are vaccinated $(V)$ before being hospitalized and enter directly into the "vaccinated" compartment.

In Figure 1, the arrows indicate the inflows and outflows for each of the five states in the two model components, with their corresponding rates. The rate of hospital admissions is $\Lambda$, with corresponding fractions of patients admitted with CA-MRSA colonization, CA-MRSA infection, HA-MRSA colonization, and HA-MRSA infection denoted by $\lambda_{C C(V)}, \lambda_{I C(V)}, \lambda_{C H(V)}$, and $\lambda_{I H(V)}$, respectively (unvaccinated/vaccinated). $\lambda_{S V}$ represents the fraction of susceptible patients who received the vaccine prior to hospital admission. The mean length of hospital stay is defined as $1 / \gamma_{S}, 1 / \gamma_{C}$, and $1 / \gamma_{H}$, for susceptible patients, patients colonized with CA-MRSA, and patients colonized with HA-MRSA, respectively.

For both the "Unvaccinated" and "Vaccinated" compartments here, the forces of infection (e.g., the per susceptible risk of infection) for HA-MRSA $\left(F_{H}\right)$ and CA-MRSA $\left(F_{C}\right)$, respectively, are governed by the following expressions:

$$
\begin{aligned}
& F_{H}=\beta_{C H}(C H+C H V) / N+\beta_{I H}(I H+I H V) / N \\
& F_{C}=\beta_{C C}(C C+C C V) / N+\beta_{I C}(I C+I C V) / N
\end{aligned}
$$

Here, $\beta_{C C}, \beta_{I C}, \beta_{C H}$, and $\beta_{I H}$ denote the effective transmission rates to susceptible patients from patients with CA-MRSA colonization, CA-MRSA infection, HA-MRSA colonization, or HA-MRSA infection, respectively. $C C(V)$, $I C(V), C H(V)$, and $I H(V)$ denote the number of patients 
with CA-MRSA colonization, CA-MRSA infection, HAMRSA colonization, or HA-MRSA infection, unvaccinated and vaccinated, respectively $N$ denotes the total hospital patient population. Transmission within the hospital can occur as a result of direct contact with patients or contaminated fomites and Health Care Workers (HCW).

In this context, the HA- and CA-MRSA colonization rates of susceptible unvaccinated $(S)$ patients are given by: $(1-\eta) F_{H}$ and $(1-\eta) F_{C}$, respectively, where $\eta$ denotes the hospital hygiene compliance level. For vaccinated susceptible $(S V)$ patients, we can also include the potential for vaccine efficacy against carriage acquisition, $v_{C C}$ and $v_{C H}$ - and thus the corresponding colonization terms can be amended as follows $\left(1-v_{C H}\right)(1-\eta) F_{H}$ and $\left(1-v_{C C}\right)$ $(1-\eta) F_{C}$, respectively.

The infection rates in colonized patients with CAMRSA and patients with HA-MRSA are $\phi_{C}$ and $\phi_{H}$, respectively. For the vaccinated population, we consider the vaccine efficacy against infection $\left(v_{I C}\right.$ and $\left.v_{I H}\right)$ by introducing reduction factors in the corresponding infection rates as $\left(1-v_{I H}\right) \phi_{H}$ and $\left(1-v_{I C}\right) \phi_{C}$, respectively.

As a starting point, we considered similar vaccine efficacy against carriage or infection for both CA-MRSA and HA-MRSA, i.e. $v_{C C}=v_{C H}=v_{C}$ and $v_{I C}=v_{I H}=v_{I}$.

The cure rates of patients with CA-MRSA infection and HA-MRSA infection are $\tau_{C}$ and $\tau_{H}$, while the death rates of these patients are $\delta_{C}$ and $\delta_{H}$, respectively. The decolonization rates of patients with CA-MRSA coloni zation and HA-MRSA colonization are $\alpha_{C}$ and $\alpha_{H}$, respectively. The mathematical model formulation is standard for mechanistic deterministic compartmental models of disease spread in mathematical epidemiology, based on the law of mass action and resulting in a characteristic set of non-linear ordinary differential equations [31] For each of the ten distinctive model states illustrated by the different compartments in Figure 1, the rate of change over time equals the specific inflow minus the corresponding outflow, characterized by the rates indicated on the corresponding arrows in the model schematic in Figure 1, with related definitions and mathematical details given in Tables 1 and 2. The total hospital patient population $N$ here is maintained constant at full hospital occupancy ( $N=400$ patients) by varying the hospital admission rate $\Lambda$ so that the total mass balance is properly closed.

\section{Model parameters}

In this analysis, we are interested in outcomes while varying the following input parameters outlined in Table 2:

1. Hospital hygiene compliance with measures aimed at reducing MRSA transmission (e.g. hand hygiene, wearing gloves), governed by the hygiene compliance model parameter $\eta$. Compliance level may vary between zero (corresponding to $0 \%$ compliance, $\eta=0$ ) and one (corresponding to $100 \%$ compliance, $\eta=1$ ).

2. Hospital adherence and compliance to patients' screening and isolation for MRSA colonization: finding the carriers and placing them on contact precautions within the hospital, governed by the screening efficacy model parameter $s$. It may vary between 0\% $(s=0)$ : no MRSA carrier is placed on contact precaution and 100\% $(s=1)$ : all MRSA carriers are placed on the same contact precautions as the MRSA-infected patients.

3. Hospital decolonization of colonized patients: identifying the carriers and applying decolonization regimens within the hospital, governed by the decolonization efficacy model parameter $d$. It may vary between $0 \%\left(d_{=0}\right)$ : no successful decolonization, and $100 \%\left(d_{=1}\right): 100 \%$ of patients decolonized daily return to susceptible status. Various combinations of control strategies 1-3 can be considered to mimic implementation of bundle measures in the hospital.

4. Vaccine coverage: fraction of admitted patients who got vaccinated prior to admission to the hospital, governed by the vaccine coverage model parameter c. It can be varied between $0 \%\left(c_{=0}\right)$ and $100 \%\left(c_{=1}\right)$.

5. Vaccine efficacy against colonization: vaccineinduced protection against carriage acquisition (reduced risk of getting colonized once vaccinated), governed by the vaccine efficacy model parameters $v_{C C}=v_{C H}$ ). This can vary between $0 \%$ (same risk of carriage acquisition in unvaccinated and vaccinated patients, $v_{C C}=v_{C H}=0$ ) and $100 \%$ (none of the vaccinated patients become colonized, in the hospital $v_{C C}=v_{C H}=1$ ).

6. Vaccine efficacy against infection if colonized: vaccine-induced protection against developing MRSA infection in MRSA-colonized vaccinated patients (reduced risk of developing MRSA infection in MRSA vaccinated colonized patients), governed by the vaccine efficacy model parameter $\left.v_{I C}=v_{I H}\right)$. This can vary between 0\% (vaccinated MRSAcolonized patients develop MRSA infection at the same rate as non-vaccinated MRSA-colonized patients, $v_{I C}=v_{I H}=0$ ) and $100 \%$ (no vaccinated MRSA-colonized patients develop infection, $\left.v_{I C}=v_{I H}=1\right)$.

In this paper, we are primarily interested in model-based projections of MRSA infection reduction in a hospital setting following implementation of different hygiene prevention measures and potential $S$. aureus vaccination scenarios. This can be achieved by varying the values of the six model parameters between $0 \%$ and 100\% accordingly. 
Table 2 Mathematical representation of different control strategies and related parameters in the current modelling framework

\begin{tabular}{|c|c|c|}
\hline & Unvaccinated & Vaccinated \\
\hline \multirow[t]{7}{*}{ Colonization rate CA-MRSA } & \multirow[t]{2}{*}{$(1-\eta) F_{C,} 0 \leq \eta \leq 1$} & $\left(1-v_{C C}\right)(1-\eta) F_{C 1} 0 \leq v_{C C} \leq 1$ \\
\hline & & $v_{C C}:$ Vaccine efficacy against CA-MRSA colonization \\
\hline & \multicolumn{2}{|c|}{$\eta$ : Hygiene compliance parameter } \\
\hline & \multicolumn{2}{|c|}{$F_{C}=\beta_{C C}(C C+C C V) / N+\beta_{I C}(I C+I C V) / N$} \\
\hline & \multirow{2}{*}{\multicolumn{2}{|c|}{$\begin{array}{l}\beta_{C C}=\beta_{C C}^{\text {baseline }}-s\left(\beta_{C C}^{\text {baseline }}-\beta_{I C}^{\text {baseline }}\right), \quad 0 \leq s \leq 1 \\
\beta_{I C}=\beta_{I C}^{\text {baseline }}\end{array}$}} \\
\hline & & \\
\hline & \multicolumn{2}{|c|}{$s$ : Screening efficacy parameter } \\
\hline \multirow[t]{5}{*}{ Colonization rate HA-MRSA } & $(1-\eta) F_{H}, 0 \leq \eta \leq 1$ & $\left(1-v_{C H}\right)(1-\eta) F_{H^{\prime}} 0 \leq v_{C H} \leq 1$ \\
\hline & & $V_{C H}:$ Vaccine efficacy against HA-MRSA colonization \\
\hline & \multirow{3}{*}{\multicolumn{2}{|c|}{$\begin{array}{l}F_{H}=\beta_{C H}\left(C H+C H V / N+\beta_{I H}(I H+I H V) / N\right. \\
\beta_{C H}=\beta_{C H}^{\text {baseline }}-s\left(\beta_{C H}^{\text {baseline }}-\beta_{I H}^{\text {baseline }}\right), 0 \leq s \leq 1 \\
\beta_{I H}=\beta_{I H}^{\text {baseline }}\end{array}$}} \\
\hline & & \\
\hline & & \\
\hline \multirow[t]{4}{*}{ Decolonization rate CA-MRSA } & $a_{C}$ & $a_{c}^{*}$ \\
\hline & $a_{c}^{*}=a_{c}$ & \\
\hline & $a_{C}=d \gamma_{C C,} 0 \leq d \leq 1$ & \\
\hline & \multicolumn{2}{|c|}{$d$ : Decolonization efficacy parameter } \\
\hline \multirow[t]{3}{*}{ Decolonization rate HA-MRSA } & $a_{H}$ & $a_{H}^{*}$ \\
\hline & \multicolumn{2}{|l|}{$a_{H}^{*}=a_{H}$} \\
\hline & \multicolumn{2}{|l|}{$a_{H}=d \gamma_{C H}, 0 \leq d \leq 1$} \\
\hline \multirow[t]{2}{*}{ Rate of progression to CA-MRSA infection after colonization } & \multirow[t]{2}{*}{$\varphi_{C}$} & $\left(1-v_{I C}\right) \varphi_{C,} 0 \leq v_{I C} \leq 1$ \\
\hline & & $V_{K}:$ Vaccine efficacy against CA-MRSA infection \\
\hline \multirow[t]{2}{*}{ Rate of progression to HA-MRSA infection after colonization } & \multirow[t]{2}{*}{$\varphi_{H}$} & $\left(1-v_{H H}\right) \varphi_{H_{H}} 0 \leq v_{H_{H}} \leq 1$ \\
\hline & & $V_{\mathbb{H}}:$ Vaccine efficacy against HA-MRSA infection \\
\hline \multirow[t]{19}{*}{ Vaccination prior to hospital admission } & \multicolumn{2}{|c|}{ Fraction of vaccinated susceptible flowing into the hospital: } \\
\hline & \multicolumn{2}{|c|}{$\lambda_{S V}=c\left(1-\lambda_{C C}^{\text {baseline }}-\lambda_{C H}^{\text {baseline }}-\lambda_{I C}^{\text {baseline }}-\lambda_{I H}^{\text {baseline }}\right)$} \\
\hline & \multicolumn{2}{|c|}{ Fraction of vaccinated colonized CA-MRSA flowing into the hospital } \\
\hline & \multicolumn{2}{|c|}{$\lambda_{C C V}=c \lambda_{C \subset}^{\text {baseline }}$} \\
\hline & \multicolumn{2}{|c|}{ Fraction of vaccinated colonized HA-MRSA flowing into the hospital: } \\
\hline & \multicolumn{2}{|c|}{$\lambda_{C H V}=C \lambda_{C H}^{\text {baseline }}$} \\
\hline & \multicolumn{2}{|c|}{ Fraction of vaccinated infected CA-MRSA flowing into the hospital: } \\
\hline & \multicolumn{2}{|c|}{$\lambda_{I C V}=c \lambda_{I C}^{\text {baseline }}$} \\
\hline & Fraction of vaccinate & nfected HA-MRSA flowing into the hospital: \\
\hline & $\lambda_{\mathbb{H V}}=C \lambda_{\mathbb{H}}^{\text {baseline }}$ & \\
\hline & Fraction of unvaccina & d colonized CA-MRSA flowing into the hospital: \\
\hline & $\lambda_{C C}=(1-c) \lambda_{C C}^{\text {baseline }}$ & \\
\hline & Fraction of unvaccin & d colonized HA-MRSA flowing into the hospital: \\
\hline & $\lambda_{C H}=(1-C) \lambda_{C H}^{\text {baseline }}$ & \\
\hline & Fraction of unvaccina & d infected CA-MRSA flowing into the hospital: \\
\hline & $\lambda_{/ C}=(1-c) \lambda_{/ C}^{\text {baseline }}$ & \\
\hline & Fraction of unvaccin & d infected HA-MRSA flowing into the hospital: \\
\hline & $\lambda_{\mathbb{H}}=(1-c) \lambda_{\mathbb{H}}^{\text {baseline }}$ & \\
\hline & c: Vaccine coverage & arameter, $0 \leq c \leq 1$ \\
\hline
\end{tabular}


A certain level of compliance with hygiene prevention measures (\% hand hygiene +/-\% screening +/-\% decolonization) will presumably result in a consequent projected reduction in the number of MRSA infections. Upon potential implementation of vaccination, in addition to hygiene prevention measures, a further reduction in the rate of infection is conceivable. The projected magnitude of this additional reduction will depend on the existing hygiene prevention measures, and compliance to them. An intuitive argument could be made that if compliance with hygiene prevention measures is sufficiently high, there may be less potential for additional reduction of MRSA infection via vaccination with a $S$. aureus vaccine; from both a public health as well as a vaccine development perspective, however, more evidence-based answers and estimates are needed.

In-line with the primary purpose of this work, for all the outcomes presented here, with the exception of the six parameters described above, all the other model parameters were kept fixed at the baseline values from the original publications $[23,24]$, which we employ here at face value. These baseline parameter values, as described in [23,24], are summarized here in Table 1 for completeness and clarity. Although various other sensitivity analyses could be carried out, in order to maintain tractability, in the present analysis we chose to restrict the scope and focus on the model parameters controlling the different interventions. Sensitivity analyses with respect to other model input parameters are presented in the original paper by D'Agata et al. [23], and more technical and mathematical details are discussed in the paper published by Webb et al. [24]. All our numerical simulations are carried out in MATLAB R2010b The MathWorks, Inc., Natick, Massachusetts, United States.

\section{Results}

All the results presented here are at steady-state, as transient regimes are short-lived i.e. a few months (see also
Figure 2 in [23]) and not of main interest for longer term infection control policies in the hospital.

The key terms employed in this section are summarized for clarity in Table 3 .

The impact of hygiene prevention measures on MRSA infection, as function of compliance, is illustrated in Figure 2. All the percentage reductions in MRSA infection prevalence shown in this figure were computed relative to a base case corresponding to no hygiene prevention measures in place. With 50\% screening compliance and 50\% efficacy of decolonization, a model-projected reduction of $48 \%$ can be achieved even at $0 \%$ hygiene compliance levels; this reduction can go up to $72 \%$ under similar conditions, assuming $100 \%$ hygiene compliance levels. At 100\% screening compliance and 50\% efficacy of decolonization, a reduction of $64 \%$ is projected by the model at $0 \%$ hygiene compliance level. The maximum reduction projected by this model in a best case scenario (100\% screening compliance and 100\% efficacy of decolonization) is $76 \%$ (Figure 2).

Figure 3 illustrates the relative (\%) additional reduction in MRSA infection prevalence due to $S$. aureus vaccination, as a function of the hygiene compliance levels, assuming hygiene measures are in place, for a vaccine coverage fixed at 25\% (Figure 3, top panels) and 75\% (Figure 3, bottom panels) respectively. Each percentage reduction in MRSA infection prevalence shown in this figure was computed relative to a corresponding base case without vaccination. Hence, the reductions reported in this figure reflect the potential added impact of vaccination on top of other existing preventative measures, for various case scenarios.

We would like to note here that due to the inherent high-dimensional nature of this analysis, based on varying six model parameters, there are many different possibilities for visualizing and reporting the outcomes. The figures shown here, while non-exhaustive, are intended to reflect results in-line with the primary scope of this work.

As an illustrative example, consider for instance an average case scenario for preventative measures in place, with $50 \%$ decolonization efficacy, 50\% screening compliance,
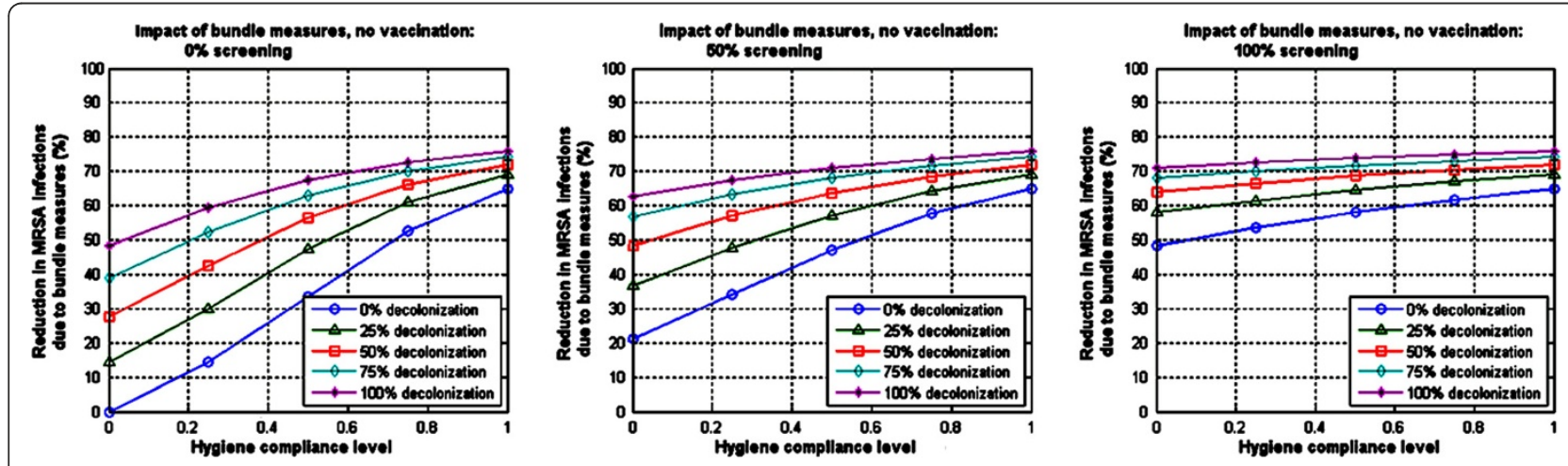

Figure 2 Illustration of model-projected impact of bundle measures only (no vaccination) on MRSA infection prevalence reduction. All results shown here are at steady-state. 
Table 3 Definition of key terms employed throughout the analyses

\begin{tabular}{ll}
\hline Term & Definition \\
\hline Hygiene compliance level & Hospital compliance with hand washing, wearing gloves, etc., varying between 0 (corresponding to hygiene \\
& compliance parameter $\eta=0$ ) and 1 (corresponding to hygiene compliance parameter $\eta=1$ ). \\
Screening & Identify the carriers and place them on contact precautions within the hospital. " $\mathrm{x} \%$ screening" interpretation \\
& here: hospital compliance to finding and placing carriers on contact precautions; varies between $0 \%$ \\
& (corresponding to screening efficacy parameter $s=0$ ): no MRSA carrier is placed on contact precaution and \\
& $100 \%$ (corresponding to screening efficacy parameter $s=1$ ): all MRSA carriers are placed on the same contact \\
& precautions as the MRSA-infected patients.
\end{tabular}

Decolonization

Identify the carriers and apply decolonization regimens within the hospital. "x\% decolonization" interpretation here: actual efficacy of decolonization protocol, varying between $0 \%$ (corresponding to decolonization efficacy parameter $d=0$ ): no successful decolonization, and 100\% (corresponding to decolonization efficacy parameter $d=1$ ): $100 \%$ of patients decolonized daily return to susceptible status Vaccine efficacy against
colonization

Vaccine efficacy against infection if colonized

Vaccine coverage
Vaccine-induced protection against carriage acquisition (reduced risk of colonization once vaccinated); can be varied between $0 \%$ (corresponding to vaccine efficacy parameter $v_{C C}=v_{C H}=0$ ) and $100 \%$ (corresponding to vaccine efficacy parameter $\left.v_{C C}=v_{C H}=1\right)$. Here we assumed for simplicity that $v_{C C}=v_{C H}$.

Vaccine-induced protection against developing actual MRSA infection in MRSA-colonized patients (lower risk of developing MRSA infection in MRSA vaccinated colonized patients); can be varied between 0\% (corresponding to vaccine efficacy parameter $v_{I C}=v_{I H}=0$ ) and $100 \%$ (corresponding to vaccine efficacy parameter $v_{I C}=v_{I H}=1$ ). Here we assumed for simplicity that $v_{I C}=v_{I H}$.

Fraction of admitted patients who got vaccinated prior to admission to the hospital. " $x \%$ vaccine coverage" interpretation here: $x \%$ of the patients admitted daily into the hospital have been vaccinated prior to admission; can be varied between 0\% (corresponding to vaccine coverage parameter $c=0$ ) and 100\% (corresponding to vaccine coverage parameter $c=1$ ). and $50 \%$ hygiene compliance level. In such a case, for a vaccination case scenario with $25 \%$ vaccine coverage and $75 \%$ vaccine efficacy against infection, the model-estimated additional reduction in MRSA infection prevalence due to vaccination is $12 \%$ (Figure 3 [top left]), assuming 0\% vaccine efficacy against colonization. Alternatively, for the same average case, with vaccine coverage of $75 \%$, and vaccine efficacy against infection of $75 \%$, the model-estimated additional reduction in MRSA infections due to vaccination is $37 \%$ (Figure 3 [bottom left]), assuming $0 \%$ vaccine efficacy against colonization. Higher additional reductions could be achieved if the vaccine had protective effects against carriage acquisition as well (Figure 3 [top right] and [bottom right]).

Figures 4 and 5 show the annual number of vaccine doses in each of these case scenarios (right panels) and the corresponding number of MRSA infections averted (left panels) due to vaccination, assuming one vaccine dose per patient. In our "average" preventative measure case discussed above, for a vaccination case scenario with $25 \%$ vaccine coverage and $75 \%$ vaccine efficacy against infection, the model estimates that at 52 additional cases of MRSA infection could be averted annually in a hospital setting with 6815 doses of vaccine (Figure 4 [top]), assuming $0 \%$ vaccine efficacy against colonization. Alternatively, with vaccine coverage of $75 \%$, and vaccine efficacy against infection still at $75 \%$, the model estimates that 158 additional cases of MRSA infection could be averted annually in a hospital setting with 20680 doses of vaccine (Figure 5 [top]), assuming $0 \%$ vaccine efficacy against colonization. Additional benefits that could be obtained if a potential vaccine had an impact on colonization as well are illustrated in the corresponding plots in Figure 4 [bottom] and Figure 5 [bottom], for a case scenario where a vaccine would have $50 \%$ efficacy against colonization.

\section{Discussion}

In this paper, we explore for the first time whether hypothetical $S$. aureus vaccination in addition to other preventative measures may have the potential to further reduce MRSA infection in a given hospital setting and we attempt to provide estimates of the potential magnitude of such reductions under various scenarios. To this end, we employ a versatile dynamic transmission modelling framework. Model-based projections indicate that even with implementation of other hygiene prevention measures, $S$. aureus vaccination could potentially provide additional reduction of MRSA infection in a hospital setting. Vaccine coverage and vaccine efficacy are key factors that would impact the magnitude of this reduction. To our knowledge, this is the first study to assess potential of a $S$. aureus vaccine to yield additional MRSA infection reduction in a hospital setting, on top of other preventative measures that already proved efficient.

Model-based simulations illustrate how different levels of reduction of MRSA infection can be achieved at the hospital level with various combinations of interventions such as hospital hygiene, decolonization and contact precautions. These interventions have been shown to be effective in decreasing the spread of MRSA in hospitals $[10-15,18]$, but their impact is variable, depending on the level of compliance. Non-compliance with hand hygiene is recognized as the most important modifiable 


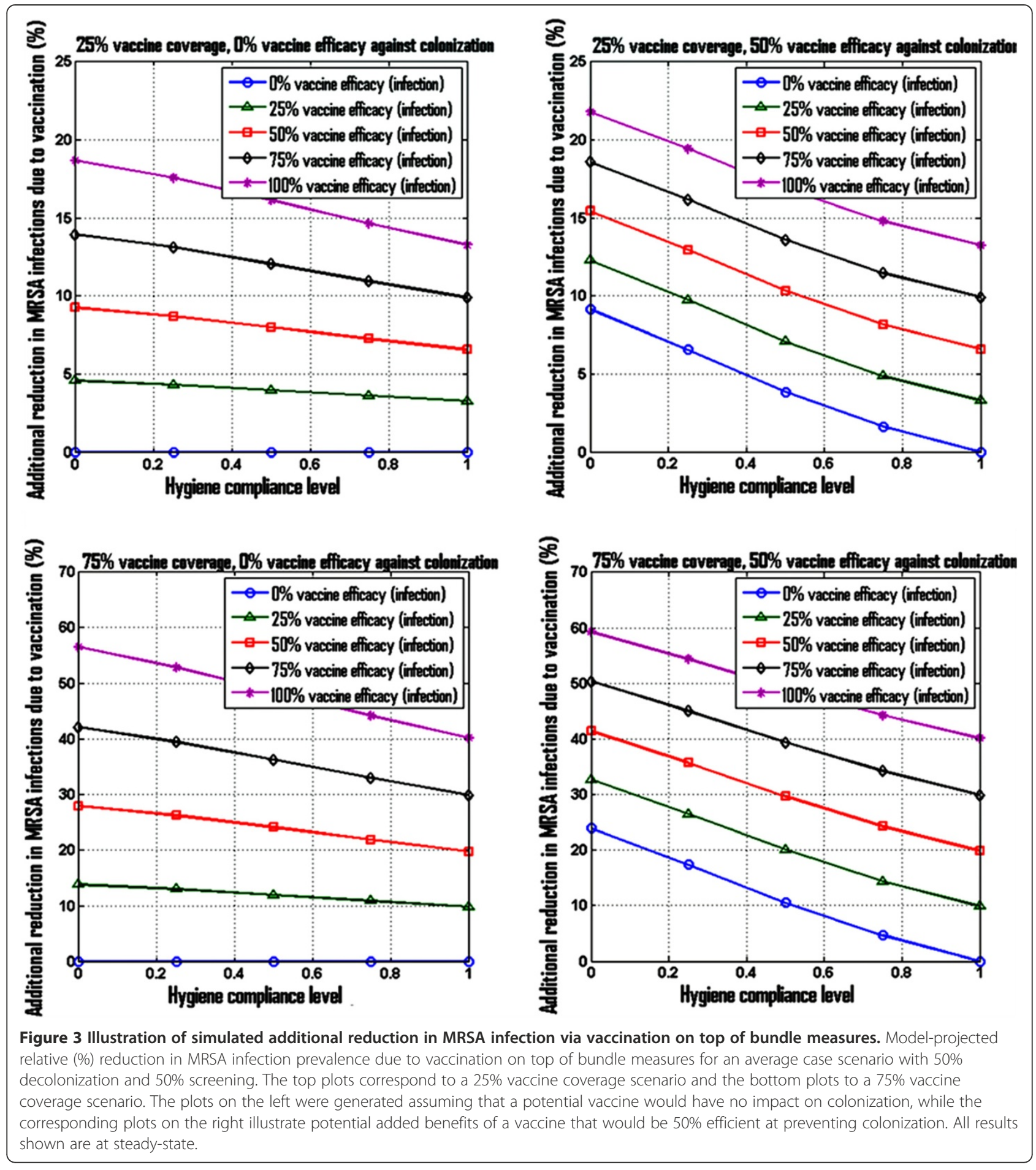

cause of healthcare-acquired infections [32,33]. Despite existing guidelines, rates of compliance with hand hygiene recommendations remain low in hospitals. The Center for Disease Control and Prevention reported that among $\mathrm{HCW}$, the national average rate of compliance with recommended hand hygiene procedures in the US was approximately $40 \%$ in 2002 [32]. However, higher compliance with hygiene recommendations is reported in different hospital settings, reaching for instance $90 \%$ in the Duke Hospital network in 2010 [34]. According to a recent comprehensive review of literature published as a Cochrane Review [35], the quality of intervention studies intended to evaluate and to improve hand hygiene compliance remains low. 

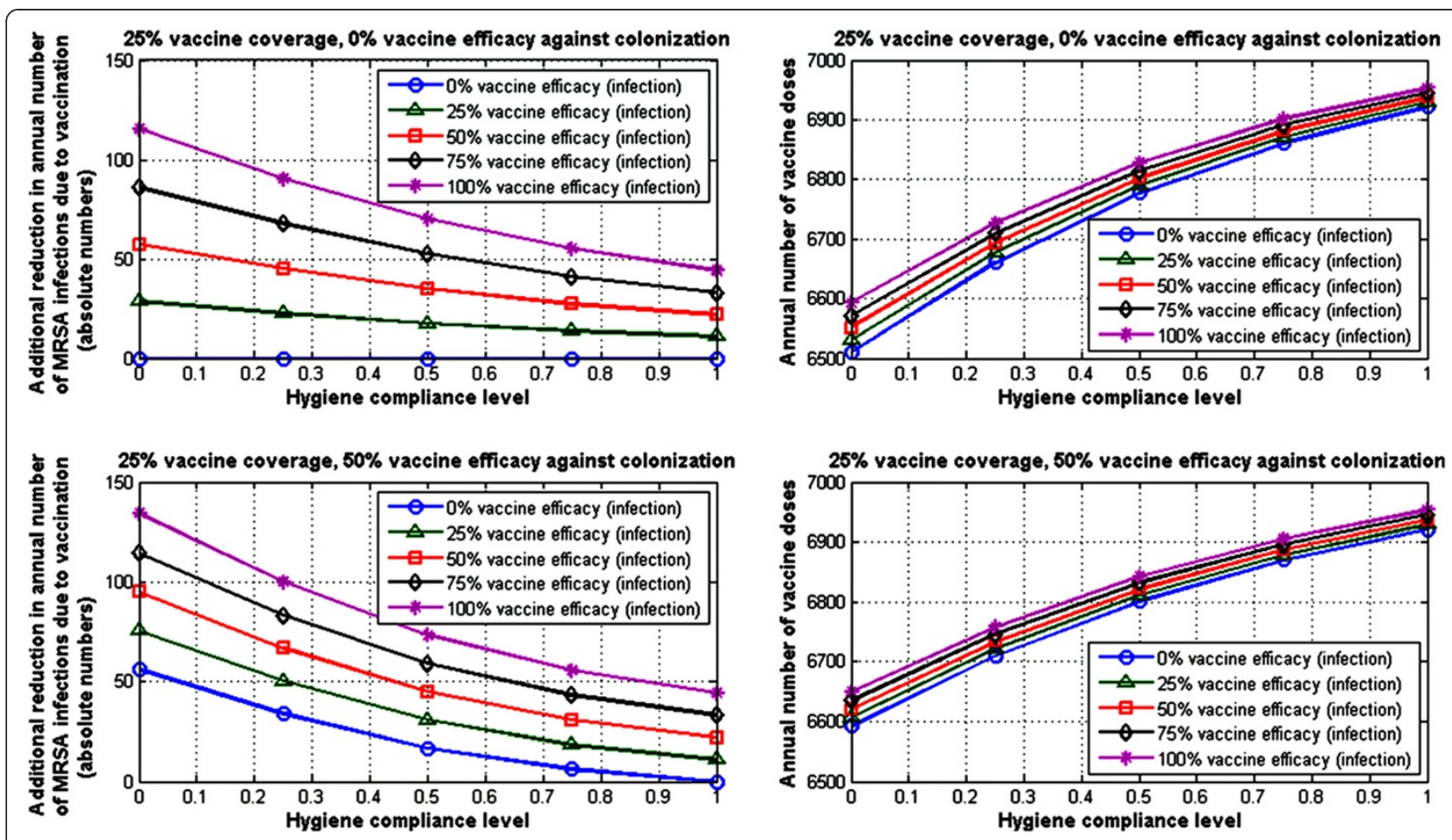

Figure 4 Model-based projections, 25\% vaccine coverage: annual number of cases averted and corresponding number of doses. Left panel: model-based projected reduction in the annual number of MRSA infections due to vaccination at $25 \%$ vaccine coverage on top of bundle measures, as a function of the hygiene compliance level, for an average case scenario with $50 \%$ decolonization and $50 \%$ screening. Right panel: annual number of vaccine doses necessary to achieve the corresponding levels of MRSA infection reduction, assuming one dose per patient. All results are shown at steady-state. The slight variations in the number of doses here for the same level of vaccine coverage reflect the corresponding differences in the number of daily admissions, which is allowed to vary in each instance to ensure full hospital occupancy at all times.

Robicsek et al. [14] reported that the implementation of universal MRSA surveillance (about 90\%, screening) plus decolonization and isolation in a 3-hospital network (with about 850 beds) led to a $69.6 \%$ reduction in the number of MRSA infections compared to baseline (prior to introduction of active surveillance) [14]. Using similar measures, Jain and colleagues [18] reported a decrease of $62 \%$ in the number of healthcare-associated MRSA infections in ICUs belonging to Veterans Affairs hospitals. Our model projections estimate reductions in MRSA infection prevalence of $60-75 \%$ under related conditions.

However, patient protection against MRSA infection based on such interventions only is likely to be limited to the hospitalization period and up to 30 days after hospital discharge [14], while a vaccine may potentially provide longer term protection (e.g. several months [36]). This is an important aspect since patients who are more susceptible to HA-MRSA and CA-MRSA hospital infections [37-39] may also require repeated episodes of hospitalization [40], which would presumably be reduced through the implementation of MRSA preventative measures. Recently, Huang et al. [40] reported the results of a retrospective cohort study evaluating the risk of MRSA infection, hospitalization and death after hospital discharge among high-risk patients who had been newly identified as harbouring MRSA. Between January 1991 and December 2003, 591 new MRSA carriers were identified: 23\% colonized and $77 \%$ infected, at the time of detection. In the year following the identification of the carrier state, 196 patients developed 317 MRSA infections. Of these cases of MRSA infections, 26\% involved bacteraemia and 17\% led to MRSA-attributable death [40].

Here, we emphasize the fact that the model considered in this paper is for MRSA only, focusing on the reduction in MRSA infection in a hospital setting, and does not include MSSA. MSSA continues to represent a substantial proportion of all $S$. aureus infections in hospitals [1], and future expansions of the model to include MSSA should be considered if similar MSSA hospital transmission data become available. One of the reasons for building a vaccination component starting from an existing versatile model [23] was the fact that baseline values for the model parameters were coherently provided in a given hospital setting. Generally, model parameters (such as transmission and progression rates) need to be estimated by calibrating the model against data. For such models to be useful in practice, the level of model complexity needs to be supported by available 

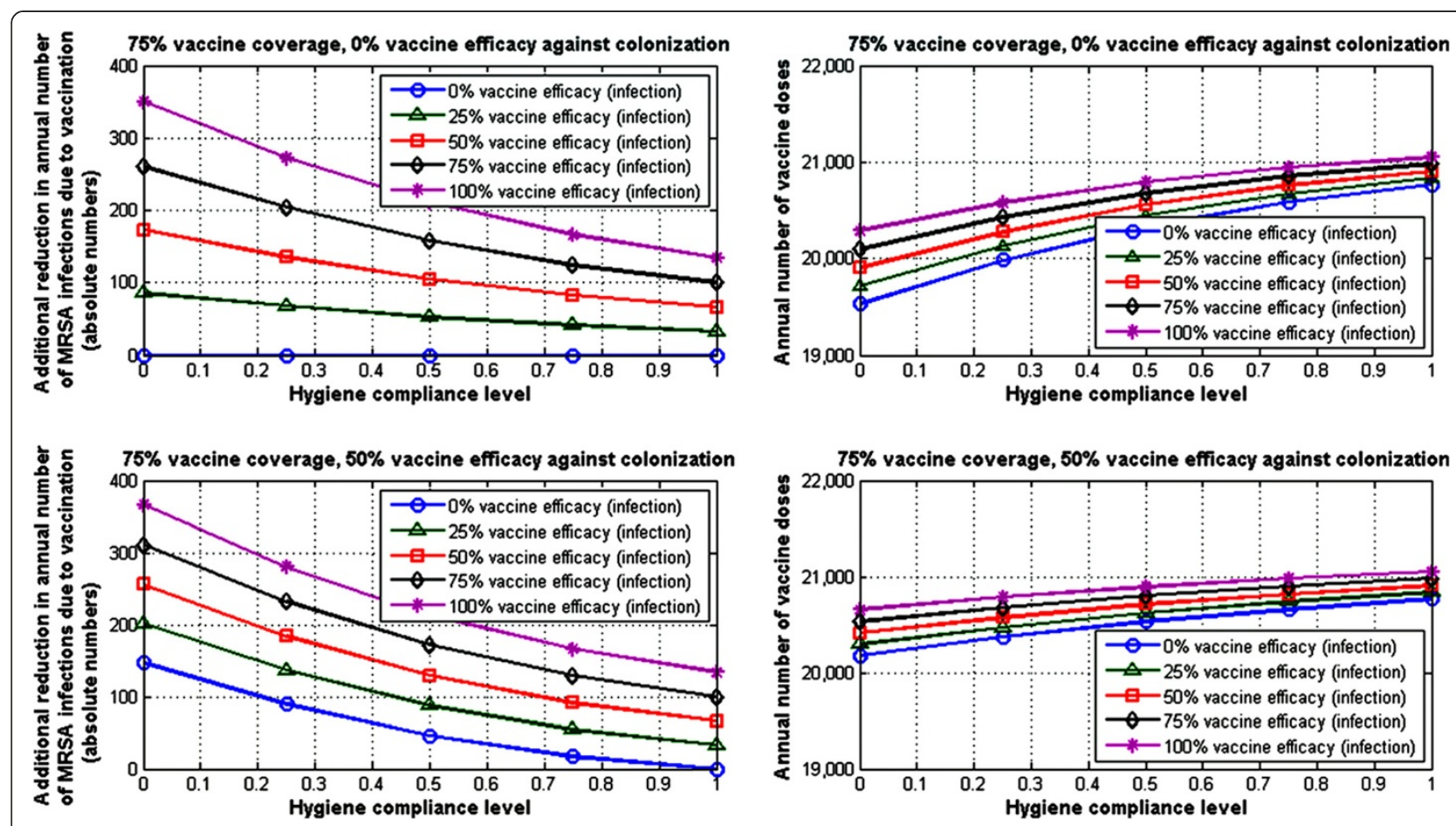

Figure 5 Model-based projections, 75\% vaccine coverage: annual number of cases averted and corresponding number of doses. Left panel: model-based projected reduction in the annual number of MRSA infections due to vaccination at $75 \%$ vaccine coverage on top of bundle measures, as a function of the hygiene compliance level for an average case scenario with 50\% decolonization and 50\% screening. Right panel: annual number of vaccine doses necessary to achieve the corresponding levels of MRSA infection reduction, assuming one dose per patient. All results are shown at steady-state. The slight variations in the number of doses here for the same level of vaccine coverage reflect the corresponding differences in the number of daily admissions, which is allowed to vary in each instance to ensure full hospital occupancy at all times.

data. Here, we did not have data available for MSSA in this hospital setting. Also, following the original model in $[23,24]$, in this simplified modeling framework, dual CA-MRSA/HA-MRSA colonization is not explicitly considered. Information/data about co-colonization with CA-MRSA and HA-MRSA is currently largely missing, as most studies tend to look at colonization separately. Co-existence in this modeling framework is enabled via adequate steady influxes of correspondingly colonized/ infected patients into the hospital. More discussions can be found in [23,24].

We also emphasize that in practice, model-based projections may be setting/hospital-dependent, as the input parameters for the underlying baseline model may vary from one hospital setting to another. In this analysis, we employed at face-value published baseline values [23,24] for the model parameters governing infection transmission and spread in a given hospital setting. Such estimates, which are key to any subsequent model outcome, can be challenged and should ideally be obtained based on model adaptation and calibration to specific hospital settings. Rationale was provided and documented by D'Agata et al., [23], and Webb et al., [24], and here we simply accepted their framework as plausible in the given hospital setting in US and used it as a starting point for the type of analyses we were primarily interested in. To ascertain actual ranges and variability for the different input parameters (e.g., inflow of colonized/ infected patients into the hospital, transmission/progression rates) so as to guide sensitivity analyses, further studies are necessary to collect and collate data from different hospital settings reflecting inherent heterogeneities (e.g., different geographical location, different type of hospital, patient population/community served).

As expected, assumed vaccine coverage has a significant impact on the model outcomes. We varied the vaccine coverage parameter between 0 and $100 \%$, and we showed here results corresponding to low (25\%) and high (75\%) vaccine coverage, respectively, for illustrative purposes. The actual levels of coverage attainable in practice are likely to depend greatly on the type of vaccination strategy (e.g., community vaccination, vaccination of planned intervention patients) as well as hospital type (e.g., general vs. specialty). A significant percentage of hospitalizations in the US are due to interventions [41], most of which are planned [42]. This percentage is typically higher in specialty hospitals [43].Vaccinating patients prior to hospital admission may theoretically provide adequate time to elicit a protective immune response [44]. In a recent Phase IIB/III clinical trial, patients were vaccinated with a 
S. aureus candidate vaccine $14-60$ days before planned cardiovascular surgery [45].

The work we present here was not intended as exhaustive or definitive, but rather a starting point. Our goals were to propose a versatile and tractable modelling and simulation framework for evaluating the impact of combined interventions on reducing MRSA infection at the hospital level and illustrate it with concrete results we found relevant in the context as a proof of concept. The proposed framework can serve further for additional analyses (e.g., cost-effectiveness) and model adaptations (e.g., other preventative measures such as screening at admission), as well as potential comparison with other baseline models (e.g., different model parameters, simplified/other approaches, etc.).

Pending data availability, further expansions of this type of modelling framework could be considered to include a dynamic exchange and double feedback between the community and the hospital setting.

Finally, we want to acknowledge that the mechanistic deterministic modelling approach we employed here, while appealing because of its built-in tractability, is limited in the sense that everything is assumed as an average at a patient population/hospital level, with no explicit account for various sources of heterogeneity, variability and uncertainty. Particularly in smaller size hospitals, such an approach may not be ideally-suited, and alternatives such as stochastic and/or individual-based models could be considered.

\section{Conclusions}

We have shown here how adequate $S$. aureus vaccination, prior to hospital admission, has the potential to further reduce burden of MRSA infection in the hospital setting, with magnitude of additional reduction varying depending on the level of other hygiene prevention measures in place. Current prevention measures such as hand hygiene are effective at reducing MRSA infection, but compliance with these measures is critical. Therefore, it is important that these practices continue to be promoted and implemented by hospitals and healthcare workers.

\footnotetext{
Abbreviations

MRSA: Methicillin-resistant S. aureus; HA-MRSA: Healthcare-associated MRSA; CA-MRSA: Community-associated MRSA; VRE: Vancomycin-resistant enterococcus; ICUs: Intensive care units; MSSA: Methicillin sensitive S. aureus; HCW: Health care workers; CH patients: Patients colonized with HA-MRSA; CC patients: Patients colonized with CA-MRSA; IH patients: Patients infected with HA-MRSA; IC patients: Patients infected with CA-MRSA; FOl: Force of infection; S patients: Susceptible patients; SV: Vaccinated susceptible patients.
}

\section{Competing interests}

AC, CH and TVE declare they are employed by GlaxoSmithKline group of companies and own stock options of GlaxoSmithKline.

\section{Authors' contributions}

$\mathrm{CH}$ designed the appropriate modelling framework, identified the data to support the model by extensive research of the literature, written the mathematical model with vaccination, implemented it in Matlab, devised the solution procedure and ran the computer simulations, devised various sensitivity analyses to address the driving questions, and provide specific answers. TVE was involved in interpretation of the modelling results. AC was involved in the design of the model and interpretation of the results. All authors read and approved the final manuscript.

\section{Acknowledgements}

The authors thank Adriana Rusu (XPE Pharma \& Science) who provided writing services on behalf of GlaxoSmithKline Vaccines and Denis Sohy (GlaxoSmithKline Vaccines), Myriam Wilbaux and Wouter Houthoofd (XPE Pharma \& Science) for publication coordination on behalf of GlaxoSmithKline Vaccines.

\section{Financial support}

GlaxoSmithKline Biologicals SA was the funding source and supported all costs associated with the development and the publishing of the present manuscript. All authors had full access to the data and agreed with the submission of the publication.

\section{Author details}

${ }^{1}$ GlaxoSmithKline Vaccines, 2301 Renaissance Blvd Ste RN0510, King of Prussia, PA, USA. ${ }^{2}$ GlaxoSmithKline Vaccines, Wavre, Belgium.

Received: 16 July 2013 Accepted: 9 May 2014

Published: 28 May 2014

\section{References}

1. Mostofsky E, Lipsitch M, Regev-Yochay G: Is methicillin-resistant Staphylococcus aureus replacing methicillin-susceptible S. aureus? J Antimicrob Chemother 2011, 66:2199-2214

2. Lee AS, Huttner B, Harbarth S: Control of methicillin-resistant Staphylococcus aureus. Infect Dis Clin North AM 2011, 25:155-179.

3. Barrett FF, McGehee RF Jr, Finland M: Methicillin-resistant Staphylococcus aureus at Boston City Hospital. Bacteriologic and epidemiologic observations. N Engl J Med 1968, 279:441-448.

4. Carleton HA, Diep BA, Charlebois ED, Sensabaugh GF, Perdreau-Remington F: Community-adapted methicillin-resistant Staphylococcus aureus (MRSA): population dynamics of an expanding community reservoir of MRSA. J Infect Dis 2004, 190:1730-1738.

5. Fridkin SK, Hageman JC, Morrison M, Sanza LT, Como Sabetti K, Jernigan JA, Harriman K, Harrison LH, Lynfield R, Farley MM, Active Bacterial Core Surveillance Program of the Emerging Infections Program Network: Methicillin-resistant Staphylococcus aureus disease in three communities. N Engl J Med 2005, 352:1436-1444.

6. Mera RM, Suaya JA, Amrine-Madsen H, Hogea CS, Miller LA, Lu EP, Sahm DF, O'Hara P, Acosta CJ: Increasing role of Staphylococcus aureus and community-acquired methicillin-resistant Staphylococcus aureus infections in the United States: a 10-year trend of replacement and expansion. Microb Drug Resist 2011, 17:321-328.

7. European Centre for Disease Prevention and Control: Surveillance of healthcare-associated infections in Europe. Stockholm: ECDC; 2012.

8. Pearson A, Chronias A, Murray M: Voluntary and mandatory surveillance for methicillin-resistant Staphylococcus aureus (MRSA) and methicillinsusceptible S. aureus (MSSA) bacteraemia in England. J Antimicrob Chemother 2009, 64(Suppl. 1):i11-i17.

9. US Department of Health and Human Services: National Action Plan to Prevent Health Care-Associated Infections: Road Map to Elimination. 2014, [http://www.health.gov/hai/prevent_hai.asp]

10. Chaberny IF, Schwab F, Ziesing S, Suerbaum S, Gastmeier P: Impact of routine surgical ward and intensive care unit admission surveillance cultures on hospital-wide nosocomial methicillin-resistant Staphylococcus aureus infections in a university hospital: an interrupted time-series analysis. J Antimicrob Chemother 2008, 62:1422-1429.

11. Harbarth S, Fankhauser C, Schrenzel J, Christenson J, Gervaz P, BandieraClerc C, Renzi G, Vernaz N, Sax H, Pittet D: Universal screening for methicillin-resistant Staphylococcus aureus at hospital admission and nosocomial infection in surgical patients. JAMA 2008, 299:1149-1157.

12. Huang SS, Yokoe DS, Hinrichsen VL, Spurchise LS, Datta R, Miroshnik I, Platt $R$ : Impact of routine intensive care unit surveillance cultures and 
resultant barrier precautions on hospital-wide methicillin-resistant Staphylococcus aureus bacteremia. Clin Infect Dis 2006, 43:971-978.

13. Ridenour G, Lampen R, Federspiel J, Kritchevsky S, Wong E, Climo M: Selective use of intranasal mupirocin and chlorhexidine bathing and the incidence of methicillin-resistant Staphylococcus aureus colonization and infection among intensive care unit patients. Infect Control Hosp Epidemiol 2007, 28:1155-1161.

14. Robicsek A, Beaumont لL, Paule SM, Hacek DM, Thomson RB Jr, Kaul KL, King P, Peterson LR: Universal surveillance for methicillin-resistant Staphylococcus aureus in 3 affiliated hospitals. Ann Intern Med 2008, 148:409-418.

15. Walsh EE, Greene L, Kirshner R: Sustained reduction in methicillin-resistant Staphylococcus aureus wound infections after cardiothoracic surgery. Arch Intern Med 2011, 171:68-73.

16. Huskins WC, Huckabee CM, O'Grady NP, Murray P, Kopetskie H, Zimmer L, Walker ME, Sinkowitz-Cochran RL, Jernigan JA, Samore M, Wallace D, Goldmann DA, STAR*ICU Trial Investigators: Intervention to reduce transmission of resistant bacteria in intensive care. N Engl J Med 2011, 364:1407-1418.

17. Chen YC, Sheng WH, Wang JT, Chang SC, Lin HC, Tien KL, Hsu LY, Tsai KS: Effectiveness and limitations of hand hygiene promotion on decreasing healthcare-associated infections. PLoS One 2011, 6:e27163.

18. Jain R, Kralovic SM, Evans ME, Ambrose M, Simbartl LA, Obrosky DS, Render ML, Freyberg RW, Jernigan JA, Muder RR, Miller LJ, Roselle GA: Veterans Affairs initiative to prevent methicillin-resistant Staphylococcus aureus infections. N Engl J Med 2011, 364:1419-1430.

19. Proctor RA: Challenges for a universal Staphylococcus aureus vaccine. Clin Infect Dis 2012, 54:1179-1186.

20. Guggenbichler JP, Assadian O, Boeswald M, Kramer A: Incidence and clinical implication of nosocomial infections associated with implantable biomaterials - catheters, ventilator-associated pneumonia, urinary tract infections. GMS Krankenhhyg Interdiszip 2011, 6:18.

21. VacZine Analytics: New vaccines for Staphylococcus aureus: risk-based or population-based approach? 2008, [http://www.vaczine-analytics.com/ ER_April_20080412.pdf]

22. Steenhuysen J: Pfizer takes its shot at a vaccine for evasive superbug. 2013 [http:// www.reuters.com/article/2013/05/23/pfizer-vaccine-idUSL2NODK0TY20130523]

23. D'Agata EM, Webb GF, Horn MA, Moellering RC Jr, Ruan S: Modeling the invasion of community-acquired methicillin-resistant Staphylococcus aureus into hospitals. Clin Infect Dis 2009, 48:274-284.

24. Webb GF, Horn MA, D'Agata EM, Moellering RC Jr, Ruan S: Competition of hospital-acquired and community-acquired methicillin-resistant Staphylococcus aureus strains in hospitals. J Biol Dyn 2010, 4:115-129.

25. Klein EY, Sun L, Smith DL, Laxminarayan R: The Changing Epidemiology of Methicillin-Resistant Staphylococcus aureus in the United States: A National Observational Study. Am J Epidemiol 2013, 177:666-674.

26. Adegbola RA, Secka O, Lahai G, Lloyd-Evans N, Njie A, Usen S, Oluwalana C, Obaro S, Weber M, Corrah T, Mulholland K, McAdam K, Greenwood B, Milligan PJ: Elimination of Haemophilus influenzae type $b$ (Hib) disease from The Gambia after the introduction of routine immunisation with a Hib conjugate vaccine: a prospective study. Lancet 2005, 366:144-150.

27. Hammitt LL, Bruden DL, Butler JC, Baggett HC, Hurlburt DA, Reasonover A, Hennessy TW: Indirect effect of conjugate vaccine on adult carriage of Streptococcus pneumoniae: an explanation of trends in invasive pneumococcal disease. J Infect Dis 2006, 193:1487-1494.

28. Maiden MC, Ibarz-Pavón AB, Urwin R, Gray SJ, Andrews NJ, Clarke SC, Walker AM, Evans MR, Kroll JS, Neal KR, Ala'aldeen DA, Crook DW, Cann K, Harrison S, Cunningham R, Baxter D, Kaczmarski E, Maclennan J, Cameron JC, Stuart JM: Impact of meningococcal serogroup $C$ conjugate vaccines on carriage and herd immunity. J Infect Dis 2008, 197:737-743.

29. Trotter CL, McVernon J, Ramsay ME, Whitney CG, Mulholland EK, Goldblatt D, Hombach J, Kieny MP, SAGE subgroup: Optimising the use of conjugate vaccines to prevent disease caused by Haemophilus influenzae type b, Neisseria meningitidis and Streptococcus pneumoniae. Vaccine 2008, 26:4434-4445.

30. Van Effelterre T, Moore MR, Fierens F, Whitney CG, White L, Pelton SI, Hausdorff WP: A dynamic model of pneumococcal infection in the United States: Implications for prevention through vaccination. Vaccine 2010, 28:3650-3660.

31. Brauer F, Driessche P, Wu J: Mathematical Epidemiology (Lecture Notes in Mathematics/Mathematical Biosciences Subseries). Heidelberg: SpringerVerlag; 2008
32. Boyce JM, Pittet D, Healthcare Infection Control Practices Advisory Committee; HICPAC/SHEA/APIC/IDSA Hand Hygiene Task Force: Guideline for Hand Hygiene in Health-Care Settings: Recommendations of the Healthcare Infection Control Practices Advisory Committee and the HICPAC/SHEA/APIC/IDSA Hand Hygiene Task Force. MMWR Recomm Rep 2002, 51:1-45. No. RR-16.

33. Pittet D, Hugonnet $S$, Harbarth S, Mourouga P, Sauvan V, Touveneau S, Perneger TV: Effectiveness of a hospital-wide programme to improve compliance with hand hygiene. Infect Control Programme Lancet 2000, 356:1307-1312.

34. Inside Duke Medicine: Hand hygiene compliance improves with monitoring program. 2010, [http://insidedukemedicine.org/news/handhygiene-compliance-improves-with-monitoring-program/]

35. Gould DJ, Moralejo D, Drey N, Chudleigh JH: Interventions to improve hand hygiene compliance in patient care. Cochrane Database 2010, 9:CD005186

36. Shinefield H, Black S, Fattom A, Horwith G, Rasgon S, Ordonez J, Yeoh H, Law D, Robbins JB, Schneerson R, Muenz L, Fuller S, Johnson J, Fireman B, Alcorn $H$, Naso R: Use of a Staphylococcus aureus conjugate vaccine in patients receiving hemodialysis. N Engl J Med 2002, 346:491-496.

37. Kourbatova EV, Halvosa JS, King MD, Ray SM, White N, Blumberg HM: Emergence of community-associated methicillin-resistant Staphylococcus aureus USA 300 clone as a cause of health care-associated infections among patients with prosthetic joint infections. Am J Infect Control 2005, 33:385-391.

38. Paez A, Skiest D: Methicillin-resistant Staphylococcus aureus: from the hospital to the community. Curr Infect Dis Rep 2008, 10:14-21.

39. Seybold U, Kourbatova EV, Johnson JG, Halvosa SJ, Wang YF, King MD, Ray SM, Blumberg HM: Emergence of community-associated methicillinresistant Staphylococcus aureus USA300 genotype as a major cause of health care-associated blood stream infections. Clin Infect Dis 2006, 42:647-656

40. Huang SS, Hinrichsen VL, Datta R, Spurchise L, Miroshnik I, Nelson K, Platt R: Methicillin-resistant Staphylococcus aureus infection and hospitalization in high-risk patients in the year following detection. PLoS One 2011 6:e24340.

41. National Center for Health Statistics: United States, 2012: With Special Feature on Emergency Care. Hyattsville, MD, USA; 2013.

42. Ingraham AM, Cohen ME, Raval MV, Ko CY, Nathens AB: Comparison of hospital performance in emergency versus elective general surgery operations at 198 hospitals. J Am Coll Surg 2011, 212:20-28.

43. US News Health Hospitals: Hospital for Special Surgeryin New York, NY: stats and services; 2013 [http://health.usnews.com/best-hospitals/area/ny/hospitalfor-special-surgery-6212900/details]

44. Joice R, Lipsitch M: Targeting Imperfect Vaccines against Drug-Resistance Determinants: A Strategy for Countering the Rise of Drug Resistance. PLoS One 2013, 8:e68940.

45. Zorman JK, Esser M, Raedler M, Kreiswirth BN, Ala'aldeen DA, Kartsonis N, Smugar SS, Anderson AS, McNeely T, Arduino JM: Naturally occurring lgG antibody levels to the Staphylococcus aureus protein IsdB in humans. Hum Vaccin Immunother 2013, 9:1857-1864.

\section{doi:10.1186/1471-2334-14-291}

Cite this article as: Hogea et al:: A model-based analysis: what potential could there be for a $S$. aureus vaccine in a hospital setting on top of other preventative measures? BMC Infectious Diseases 2014 14:291. 